\title{
Effect of Confinement on Polarization Sign Inversion Temperature in Ferroelectric Liquid Crystal
}

\author{
S.A. RÓŻAŃSKI* \\ Stanisław Staszic State University of Applied Sciences in Piła, \\ Department of Electrical Engineering, Podchorazzych 10, 64-920 Piła, Poland \\ Doi: 10.12693/APhysPolA.139.417 \\ *e-mail: srozansk@asta-net.com.pl
}

\begin{abstract}
Dielectric spectroscopy of the ferroelectric liquid crystal S-(-)-2-methylbutyl 4- $n$-nonanoyloxy-biphenyl4'-carboxylate shows the occurrence of an anomaly in temperature dependence of the dielectric strength of the Goldstone mode related with a spontaneous polarization sign reversal present in this liquid crystal compound. Particular attention was paid to the dependence of the inversion temperature on aerosil nanoparticles density. Generally, the inversion temperature decreases with increasing aerosil density as a result of a hydrogen-bonded gel structure formed in the ferroelectric liquid crystal. Both the size effects and the interaction of the liquid crystal molecules with the aerosil surfaces play an important role.
\end{abstract}

topics: dielectric spectroscopy, ferroelectric liquid crystal, confinement, polarization sign reversal

\section{Introduction}

The polarization sign inversion caused by temperature change is an intriguing phenomenon that has so far been observed in single-component materials $[1,2]$, two-component mixtures [3-5], and polymer systems [6]. A number of theoretical models have been proposed to explain the polarization sign reversal with temperature in the smectic $\mathrm{C}^{*}$ phase $\left(\mathrm{SmC}^{*}\right)$ of ferroelectric liquid crystals (FLCs). One of them took into account the presence of two different structural conformers in FLC which at an inversion temperature $T_{\text {inv }}$ are inter-convertible through a potential barrier [7]. Another model based on Landau's theory takes into account competition between the polar and quadrupole order for a singleparticle rotational potential in which molecules rotate around a molecular long axis [8].

In derivatives of substituted 2-phenylpyrimidines, the temperature change of the sign of spontaneous polarization was explained taking into account the interplay between the chiral dipole, the steric dipole and the polarizability properties of the molecules [9]. An introduction to the rotational potential of a coupling term between the molecular dipole moment and the magnitude of the electric field makes it possible to obtain a change in the sign of spontaneous polarization under the electric field [2]. In freely suspended FLC films, the value of inversion temperature decreases linearly with the reciprocal film thickness $[10,11]$. Moreover, in the three biphenyl series of FLCs it was shown that the inversion temperature $T_{\mathrm{inv}}$ depends non-monotonously on the chain length of the alkyl end group [12]. In the binary mixture of chiral FLC and achiral dopant, the inversion temperature was very sensitive to the concentration of the dopant [4]. Additionally, with increasing concentration of siloxane-terminated smectic mesogen in a binary mixture with achiral smectic $\mathrm{C}(\mathrm{SmC})$ material, the polarization sign inversion temperature shifts towards the smectic A-smectic $\mathrm{C}^{*}(\mathrm{SmA}$ $\mathrm{SmC}^{*}$ ) phase transition [5]. Some authors show that the inversion temperature $T_{\mathrm{inv}}$ increases with the increase of the electric field amplitude [2]. However, in other works, where anomalous temperature dependence of permittivity is observed, it is suggested that $T_{\mathrm{inv}}$ does not depend on the electric field and this anomaly is not significantly affected by applying pressures below $10^{8} \mathrm{~Pa}[12]$.

The dielectric spectroscopy investigations of the biphenyl ester series of FLCs reveal the presence of the characteristic permittivity anomaly in the point of polarization sign reversal $[1,12,13]$. An important question that arises in this context is whether the inversion temperature $T_{\mathrm{inv}}$ depends on the geometric constraints. In order to clarify the answer to the above question, the dielectric measurements of FLC S-(-)-2-methylbutyl 4-n-nonanoyloxybiphenyl4'-carboxylate were carried out in a wide frequency range for different aerosil concentrations [1, 14].

In the present paper, the effect of aerosil concentration on the inversion temperature of spontaneous polarization is analyzed. 


\section{Experimental}

Broadband dielectric spectroscopy (BDS) of FLC S-(-)-2-methylbutyl 4-n-nonanoyloxy-biphenyl-4'carboxylate (IS-2424) with the phase transition temperatures sequence $\mathrm{Cr}-312.15 \mathrm{~K}-\mathrm{SmC}^{*}-$ 315.65 K - SmA 332.65 K - I was performed in the frequency range from $10^{-2} \mathrm{~Hz}$ to $10^{7} \mathrm{~Hz}$. The FLC IS-2424 possesses spontaneous polarization of about $2 \mathrm{nC} / \mathrm{cm}^{2}$ and helical pitch equal to about $3 \mu \mathrm{m}[15,16]$. A characteristic feature of this FLC is the occurrence of a polarization sign inversion at a temperature of about $292 \mathrm{~K}$. The FLC was tested both in a bulk form and as an aerosil dispersion system.

To prepare the dispersion systems, a hydrophilic aerosil 300 with a primary nanoparticle size of $7 \mathrm{~nm}$ and a characteristic surface area of $300 \mathrm{~g} / \mathrm{m}^{2}$ was used [17]. The various mixtures with aerosil density $\rho_{s}=0.025,0.05,0.08,0.15 \mathrm{~g} / \mathrm{cm}^{3}$ were prepared using a solvent method, described in [16]. To calculate the density, one applies the following relation $\rho_{s}=\frac{m_{s}}{m_{\mathrm{LC}}} \rho_{\mathrm{LC}}$, where $m_{s}$ and $m_{\mathrm{LC}}$ are the masses of aerosil and liquid crystal, and $\rho_{\mathrm{LC}} \approx 1 \mathrm{~g} / \mathrm{cm}^{3}$ is the density of liquid crystal. It should be stressed that aerosil nanoparticles are covered with hydroxyl groups that enable the formation of hydrogen bonding gel-like structures inside the FLC and cause homeotropic orientation of the molecules on their surfaces.

The complex dielectric function, namely $\varepsilon^{*}(\omega)=\varepsilon^{\prime}(\omega)-\mathrm{i} \varepsilon^{\prime \prime}(\omega)$, was measured by using a Novocontrol broadband dielectric spectrometer with a high-resolution dielectric/impedance analyzer Alpha and an active sample cell. The bulk FLC and FLC-aerosil composite were placed in a measuring capacitor with gold-plated electrodes separated by $50 \mu \mathrm{m}$ glass fibers. To improve FLC orientation, the samples were slowly cooled from the isotropic to the smectic A phase. The temperature of the samples mounted in the special holder and located in the cryostat was controlled by nitrogen gas jet. The measurements were taken every $0.5 \mathrm{~K}$ to obtain sufficient resolution near the polarization sign reversal point. The dielectric measurements were performed on cooling.

The dielectric spectra analysis was carried out using a superposition of two Havriliak-Negami functions [18] and a conductivity contribution in the form

$$
\begin{aligned}
& \varepsilon *(\omega)=\varepsilon^{\prime}(\omega)-\mathrm{i} \varepsilon^{\prime \prime}(\omega)= \\
& \varepsilon_{\infty}+\sum_{j=1}^{n} \frac{\Delta \varepsilon_{j}}{\left(1+\left(\mathrm{i} \omega \tau_{j}\right)^{1-\alpha_{j}}\right)^{\beta_{j}}}-\mathrm{i} \frac{\sigma_{0}}{\varepsilon_{0} \omega^{k}},
\end{aligned}
$$

where parameters $\alpha_{j}$ and $\beta_{j}$ define broadening and asymmetry of the $j$-th relaxation process, respectively, $\Delta \varepsilon_{j}$ is the dielectric relaxation strengths and $\tau_{j}$ - the relaxation time. The high frequency limit of the permittivity and the vacuum permittivity are denoted as $\varepsilon_{\infty}$ and $\varepsilon_{0}$, respectively, $\sigma_{0}$ is the conductivity parameter and $k$ is the fitting parameter.

\section{Results and discussion}

In the studied frequency range, several relaxation processes appear in the dielectric loss spectrum of the investigated FLC. In the SmA phase, a doubly degenerated soft mode (SM) dominates where dielectric strength increases but relaxation frequency decreases with decreasing temperature close to the $\mathrm{SmA}-\mathrm{SmC}^{*}$ phase transition. However, in the $\mathrm{SmC}^{*}$ phase, the $\mathrm{SM}$ splits into the Goldstone mode (GM) and the soft amplitude mode. The dielectric strength of the GM in the $\mathrm{SmC}^{*}$ phase is temperature independent close to the phase transition and reaches its maximum. The relaxation frequency of the SM increases but its dielectric strength in the $\mathrm{SmC}^{*}$ phase decreases with decreasing temperature.

However, in the investigated FLC, the temperature induced polarization sign reversal phenomenon appears. One of the consequences of the polarization reversal is a rapid decrease in the dielectric strength of the GM to zero at the $T_{\text {inv }}$ point, and then again, an increase to much lower values with decreasing temperature.

In the FLC-aerosil dispersion, the collective relaxation processes are significantly modified. The dielectric strength of the GM and the SM mode considerably decreases with increasing aerosil density. The GM disappears for a certain concentration of aerosil nanoparticles, but the dielectric strength of the SM becomes very low and the relaxation process itself is much blurred. Moreover, the frequency degeneracy in the $\mathrm{SmA}-\mathrm{SmC}^{*}$ phase transition is removed in the presence of the aerosil nanoparticles [19, 20].

The addition of aerosil nanoparticles to the FLC also affects processes taking place near the point of polarization sign reversal. For example, the dielectric strength of the GM decreases more strongly below than above the sign reversal point with increasing aerosil density [16].

The dielectric strength of the GM calculated on the basis of Landau's theory can be expressed [21] by

$$
\Delta \varepsilon_{G}=\frac{1}{2 \varepsilon_{0} K_{3}}\left(\frac{P_{s}}{q \theta}\right)^{2},
$$

where $P_{s}$ is the spontaneous polarization, $K_{3}$ is the twist elastic constant, $q$ is the wave vector, $\theta$ is the tilt angle, and $\varepsilon_{0}$ is the vacuum permittivity.

The change in the sign of spontaneous polarization with temperature observed in the investigated FLC can be described by a model taking into account the presence of two different structural conformers which at a certain temperature $T_{\text {inv }}$ are inter-convertible through a potential barrier $[7,22,23]$. The temperature dependence of the spontaneous polarization proposed in this model has the form

$$
P_{s}(T)=\left(\frac{P_{a}}{1+\mathrm{e}^{-\kappa}}+\frac{P_{b}}{1+\mathrm{e}^{\kappa}}\right)\left(T_{c}-T\right)^{\gamma},
$$




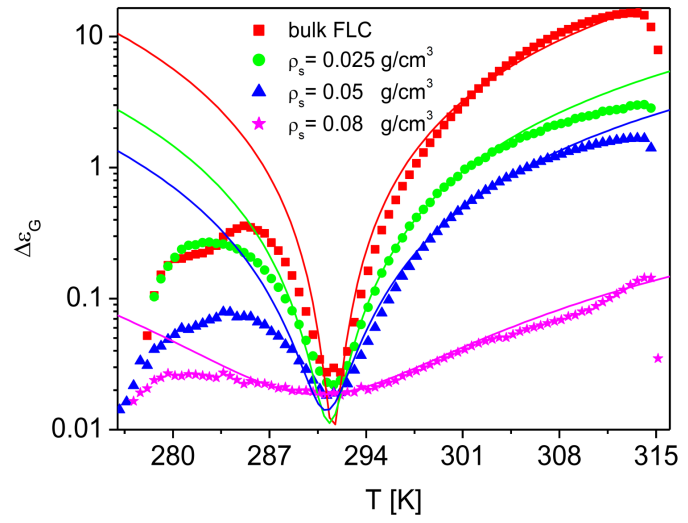

Fig. 1. Temperature dependence of the dielectric strength of the Goldstone mode for the bulk FLC and chosen densities of FLC-aerosil mixtures. The solid lines are an example of the fit of the data using (7).

where $\kappa=E_{a} /\left(k_{\mathrm{B}} T\right), E_{a}$ is the activation energy, $P_{a}$ and $P_{b}$ denote the spontaneous polarization of each species, $k_{\mathrm{B}}$ is the Boltzmann constant, $\gamma$ is the critical exponent, and $T_{c}$ is the $\mathrm{SmA}-\mathrm{SmC}^{*}$ phase transition temperature. Note that the spontaneous polarization $P_{s}\left(T_{\text {inv }}\right)=0$. Hence, from $(3)$, one can obtain

$$
P_{b}=-P_{a} \frac{1+\mathrm{e}^{\kappa_{1}}}{1+\mathrm{e}^{-\kappa_{1}}}=-P_{a} \mathrm{e}^{\kappa_{1}},
$$

where $\kappa_{1}=E_{a} /\left(k_{B} T_{\text {inv }}\right)$. Substituting (4) into (3) gives the result

$$
P_{s}(T)=P_{a}\left(\frac{\mathrm{e}^{\kappa}-\mathrm{e}^{\kappa_{1}}}{1+\mathrm{e}^{\kappa}}\right)\left(T_{c}-T\right)^{\gamma} .
$$

It should be noted that in conventional FLCs, the parameters such as $P_{s}, \theta$, and helical pitch $p=2 \pi / q$ far from the $\mathrm{SmA}-\mathrm{SmC}^{*}$ phase transition are almost temperature independent. Interestingly, in the case of the investigated FLCs, they show no anomalies at $T_{\mathrm{inv}}[24,25]$. Therefore, (5) describing the temperature dependence of the spontaneous polarization, can be simplified near $T_{\mathrm{inv}}$ as follows [22, 23]:

$$
P_{s}=\frac{P_{a} E_{a}}{k_{\mathrm{B}}}\left(\frac{1}{T}-\frac{1}{T_{\mathrm{inv}}}\right) .
$$

Substituting (6) into (2) and adding a certain constant $C$, the relationship describing the temperature dependence of the dielectric strength of the GM near the point of polarization sign reversal can be written. Namely,

$$
\Delta \varepsilon_{G}=A\left(\frac{1}{T}-\frac{1}{T_{\mathrm{inv}}}\right)^{2}+C,
$$

where $A=\frac{1}{2 \varepsilon_{0} K_{3}}\left(\frac{P_{a} E_{a}}{k_{\mathrm{B}} q \theta}\right)^{2}$.

Using (7), the dependence of $T_{\text {inv }}$ on the aerosil density was determined on the basis of fitting measurement data near the point of polarization reversal. Figure 1 presents the temperature dependence of the dielectric strength of the GM on chosen hydrophilic aerosil densities. The solid lines show
TABLE I

Fitting parameters obtained from (7) for different aerosil densities. The calculated values for the corresponding average void lengths $l_{0}$ are also provided.

\begin{tabular}{c|c|c|c|c}
\hline \hline$\rho_{s}\left[\mathrm{~g} / \mathrm{cm}^{3}\right]$ & $l_{0}[\mathrm{~nm}]$ & $A \times 10^{8}\left[\mathrm{~K}^{2}\right]$ & $T[\mathrm{~K}]$ & $C$ \\
\hline bulk FLC & bulk FLC & 2.81 & 291.60 & 0.010 \\
0.025 & 267 & 0.76 & 291.33 & 0.011 \\
0.05 & 133 & 0.38 & 291.12 & 0.014 \\
0.08 & 83 & 0.02 & 290.60 & 0.019
\end{tabular}

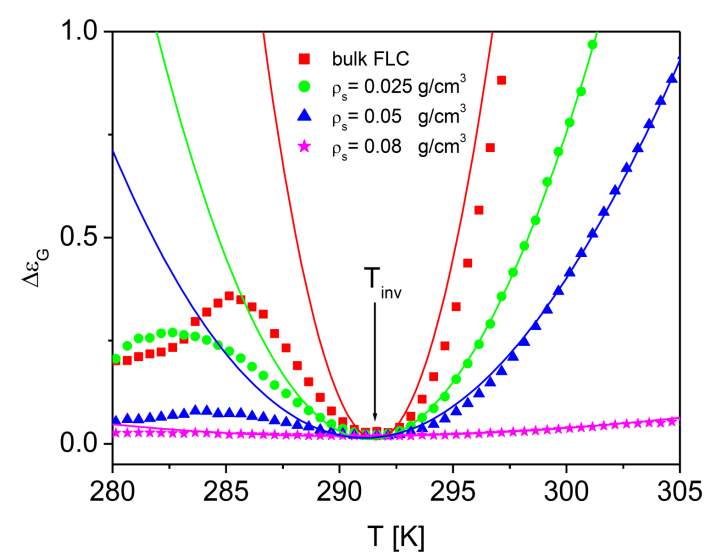

Fig. 2. Enlarged area near the point of polarization sign reversal. Each of the solid lines is the result of a fit to the experimental data using (7).

the best fit of the data using (7). Above $T_{\text {inv }}$, with increasing aerosil nanoparticles density, the amplitude of the dielectric strength gradually decreases and for higher densities excides a very low value. Also, below $T_{\text {inv }}$, the dielectric strength decreases with increasing aerosil density. The observed changes of the dielectric strength with aerosil density are caused by the modification of the FLC structure by aerosil nanoparticles and suppression of the GM.

The results obtained from fitting the data close to the point of polarization sign reversal using (7) are summarized in Table I. The average void length $l_{0}$ of the selected aerosil concentrations has also been presented and will be discussed later. The $T_{\text {inv }}$ temperature determined from fitting the data for bulk FLC agrees well with the value of $291.65 \mathrm{~K}$ obtained on the basis of the dielectric spectra inspection and observation under a polarizing microscope [1]. It should be noted that in some cases it is not just one specific temperature value, but rather the range of temperatures in which the phenomenon of spontaneous polarization reversal takes place [2].

Figure 2 shows the enlarged area of Fig. 1, very close to the point of polarization sign reversal. It can be seen that the fits with (7), expressed by solid lines, are acceptable especially for data lying above the $T_{\text {inv }}$ temperature. Below $T_{\text {inv }}$, only data lying very close to the point of polarization sign reversal are quite well matched by (7). Note also that 


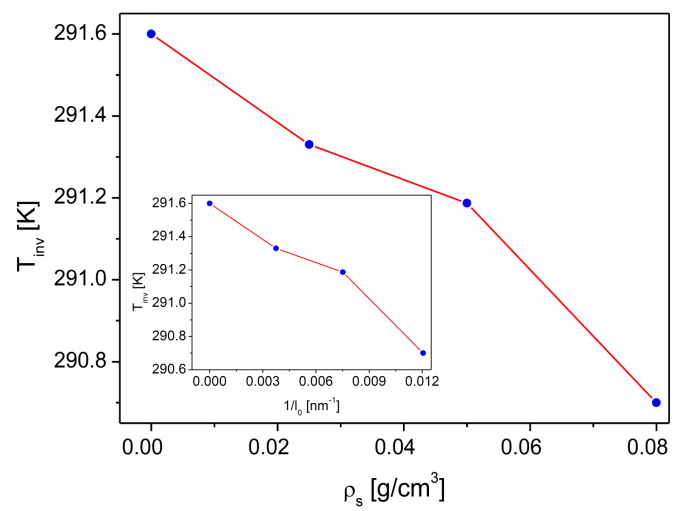

Fig. 3. The inversion temperature dependence on the aerosil density for the FLC-aerosil composite. The solid line is drawn for eye guidance only. The inset shows the dependence of the inversion temperature on the reciprocal of the average void length.

the curve for the bulk FLC data shows some deviations. Moreover, the quality of data fit using (7) depends to a large extent on the selected range of measurement points. The selected limits have been optimized to obtain the best possible match in the vicinity of $T_{\mathrm{inv}}$.

Figure 3 shows the dependence of the inversion temperature $T_{\text {inv }}$ on the aerosil density for the FLCaerosil dispersion. The data presented in Fig. 3 and summarized in Table I are obtained by fitting experimental data for bulk and every aerosil density. Considering the applied procedure for determining $T_{\mathrm{inv}}$ as appropriate in the vicinity of the polarization sign reversal, the inversion temperature determined in this way decreases with increasing aerosil nanoparticles density. The observed shift of the inversion temperature can be related with the influence of the aerosil nanoparticles on the structure of the FLC and effects on different conformers near this point $[1,16]$. For the aerosil density of about $0.025 \mathrm{~g} / \mathrm{cm}^{3}$, the temperature $T_{\text {inv }}$ slightly deviates from the linear relationship. It should be noted that for the aerosil density in the range of $0.01 \mathrm{~g} / \mathrm{cm}^{3}<\rho_{s}<0.1 \mathrm{~g} / \mathrm{cm}^{3}$, a gel-like structure is formed in liquid crystal which, due to the rearrangement of the aerosil nanoparticles network, can cause complex changes in the inversion temperature $[26,27]$.

One of the parameters characterizing the LC-aerosil dispersion is the average void length given as $l_{0}=2 /\left(a \rho_{s}\right)$, where $a=300 \mathrm{~g} / \mathrm{cm}^{3}$ for hydrophilic aerosil used in experiment and density $\rho_{s}$ was defined above (Table I). Since the parameter $l_{0}$ is inversely proportional to the aerosil density, it can be concluded that the smaller the characteristic length of the cavity, the stronger decrease of $T_{\text {inv }}$ is. In addition, it means that with the increase of the aerosil density, the structure of the liquid crystal is more strongly deformed and subjected to significant effects related to the geometric constraints. These are both interactions with the surface of the aerosil nanoparticles and dimensional effects related to the characteristic length for a given aerosil density. In the ferroelectric phase, the geometric constraints also affect the helical superstructure, leading to a modification of the Goldstone mode [19, 20].

\section{Conclusions}

The applied procedure for fitting the measurement data allowed to study the dependence of the inversion temperature on the aerosil density. The inversion temperature depends on the concentration of the aerosil nanoparticles and decreases with increase of its density.

The addition of the aerosil nanoparticles to the structure of the ferroelectric liquid crystal can lead to a modification of the potential barrier, which must be jumped by one of the two conformers probably present in the investigated liquid crystal. The effect is to lower the temperature at which the sign of spontaneous polarization changes. Furthermore, as a result of the interaction of the FLC molecules with the surface of aerosil nanoparticles, the deformation of the structure of smectic layers takes place in their vicinity, which may also affect the value of the inversion temperature. Another effect is the disturbance and deformation of the helical structure present in the FLC, which modifies the dynamics of the Goldstone mode and may lead to a shift in the polarization sign reversal temperature. Also, the presence of the hydrogen-bonded gel structure in FLC as a result of hydroxyl groups present on the surfaces of aerosil nanoparticles can lead to a modification of the rheological properties of the examined structure, which may be manifested in a decrease in the inversion temperature.

\section{Acknowledgments}

The author acknowledges the receipt of a senior postdoctoral fellowship from the Research Council of K.U. Leuven.

\section{References}

[1] S.A. Różański, J. Thoen, Liq. Cryst. 32 , 1013 (2005).

[2] Y. Mieda, H. Hoshi, Y. Takanishi, H. Takezoe, B. Žekš, Phys. Rev. E 67, 021701 (2003).

[3] H. Stegemeyer, A. Sprick, M.A. Osipov, V. Vill, H.-W. Tunger, Phys. Rev. E 51, 5721 (1995).

[4] M. Ozaki, Y. Fuwa, K. Nakayama, K. Yoshino, T. Tani, K. Fujisawa, Ferroelectrics 214, 51 (1998).

[5] D. Nonnenmacher, R.P. Lemieux, M. Osipov, F. Giesselmann, Chem. Phys. Chem. 15, 1368 (2014). 
[6] G. Scherowsky, B. Brauer, K. Grünegerg, U. Müller, L. Komitov, S.T. Lagerwall, K. Skarp, B. Stebler, Mol. Cryst. Liq. Cryst. 215, 257 (1992).

[7] J.S. Patel, J.W. Goodby, Philos. Mag. Lett. 55, 283 (1987).

[8] R. Meister, H. Stegemeyer, Ber. Bunsenges. Phys. Chem. 97, 1242 (1993).

[9] S. Saito, A. Murashiro, M. Kikuchi, D. Demus, T. Inukai, M. Neundorf, S. Diele, Ferroelectrics 147, 367 (1993).

[10] Ch. Bahr, Mol. Cryst. Liq. Cryst. 301, 313 (1997).

[11] Ch. Bahr, C.J. Booth, D. Fliegner, J.W. Goodby, Europhys. Lett. 34, 507 (1996).

[12] K. Nakao, M. Ozaki, K. Yoshino, Jpn. J. Appl. Phys. 26, 104 (1987).

[13] N. Mikami, R. Higuchi, T. Sakurai, M. Ozaki, K. Yoshino, Jpn. J. Appl. Phys. 25, L833 (1986).

[14] S.A. Różański, J. Thoen, Ferroelectrics 344, 63 (2006).

[15] S.A. Różański, in: Dielectric Properties of Liquid Crystals, Eds. E. Galewski, L. Sobczyk, Trivandrum: Transworld Research Network, 2007, p. 183.

[16] S.A. Różański, J. Thoen, J. Non-Cryst. Solids 351, 2802 (2005).
[17] AEROSIL ${ }^{\circledR}$ Fumed Silica for Cable Gels, Technical Information 1163, Evonik Industries.

[18] S. Havriliak, S. Negami, Polymer 8, 161 (1967).

[19] S.A. Różański, J. Thoen, Liq. Cryst. 32 , 331 (2005).

[20] Z. Kutnjak, S. Kralj, S. Žumer, Phys. Rev. E 66, 041702 (2002).

[21] T. Carlsson, B. Žekš, C. Filipič, A. Levstik, Phys. Rev. A 42, 877 (1990).

[22] Chang-Jae Yu, Jae-Hoon Kim, Jung-Il Jin, Sin-Doo Lee, Mol. Cryst. Liq. Cryst. 377, 373 (2002).

[23] Chang-Jae Yu, Sin-Doo Lee, Jung-Il Jin, Jae-Hoon Kim, Jpn. J. Appl. Phys. 42, 7452 (2003).

[24] R. Eidenschink, T. Geelhaar, G. Anderson, A. Dahlgren, K. Flatischler, F. Gouda, S.T. Lagerwall, K. Skarp, Ferroelectrics 84, 167 (1988).

[25] I. Musevič, I. Drevensek, R. Blinc, S. Kumar, J.W. Doane, Mol. Cryst. Liq. Cryst. 172, 217 (1989).

[26] G.S. Iannacchione, Fluid Phase Equilib. 222-223, 177 (2004).

[27] A.V. Zakharov, J. Thoen, Phys. Rev. E 69, 011704 (2004). 